\title{
Tomography by neutrino pair beam
}

\author{
Hisashi Okui*t \\ Graduate School of Science and Technology, Niigata University, 950-2181 Niigata, Japan \\ E-mail: okuidmuse.sc.niigata-u.ac.jp
}

We consider the possibility of neutrino tomography by using the neutrino pair beam. The idea of neutrino tomography is imaging of the Earth's interior structure by neutrino experiments. We assume the neutrino pair beam which has been recently proposed as an attractive neutrino source. The beam produces a huge amount of neutrino and antineutrino pairs from the circulating partially stripped ions. The beam enables us to measure the energy spectrum of the neutrino oscillation probability precisely together with a large detector. The neutrino pair beam can give a better sensitivity for probing the Earth's crust compared to current neutrino sources. Furthermore, we propose a method to reconstruct the density profile by the analytic formula of the oscillation probability that incorporates the matter effect up to the second order of perturbation.

The 4th KMI International Symposium (KMI2019)

18-20, February 2019

Nagoya, Japan

\footnotetext{
*Speaker.

${ }^{\dagger}$ This talk is based on [四]
} 


\section{Introduction}

Neutrino tomography is attractive application of neutrino physics. Tomography means getting the picture of inside of the object without destruction. In the case of neutrino tomography, we aim to obtain the image of the Earth's interior structure using neutrinos. Neutrinos can easily transmit the Earth since the neutrino interaction is very weak for energies smaller than about $\mathscr{O}(1) \mathrm{TeV}$.

There are two major methods for neutrino tomography. The first one is the absorption tomography and the second is the oscillation tomography. These methods have been reviewed in [2]. Now we focus on the neutrino oscillation tomography which is based on the matter effects of the neutrino oscillation. We can reconstruct the density profile which neutrino passed through by the energy spectrum of neutrino oscillation probability that carries the information of matter.

For simplicity, we consider two flavor case with $v_{e}$ and $v_{\mu}$. If the neutrino goes through the matter, the evolution equation is written as

$$
i \frac{d}{d x}\left(\begin{array}{c}
A_{v_{e} \rightarrow v_{e}} \\
A_{v_{e} \rightarrow v_{\mu}}
\end{array}\right)=\left[U\left(\begin{array}{cc}
0 & 0 \\
0 & \frac{\Delta m^{2}}{2 E_{v}}
\end{array}\right) U^{\dagger}+\left(\begin{array}{cc}
V_{C C}(x) & 0 \\
0 & 0
\end{array}\right)\right]\left(\begin{array}{l}
A_{v_{e} \rightarrow v_{e}} \\
A_{v_{e} \rightarrow v_{\mu}}
\end{array}\right),
$$

where $A_{v_{\alpha} \rightarrow v_{\beta}}$ is the transition amplitude of $v_{\alpha} \rightarrow v_{\beta}, U$ is the unitary mixing matrix, $\Delta m^{2} \equiv$ $m_{2}^{2}-m_{1}^{2}$ is mass squared difference, $E_{v}$ is neutrino energy. $V_{C C}(x)$ is the effective potential of the matter effect, $V_{C C}(x)=\sqrt{2} G_{F} n_{e}(x)$, where $G_{F}$ is the Fermi constant and $n_{e}(x)$ is number density of electron in matter. We assume the equality of the number density of protons and electrons due to the neutrality of matter in the Earth, $n_{e}=n_{p}$. We also apply the approximations $m_{n} \simeq m_{p} \gg m_{e}$ and $n_{n}=n_{p}$. Then the electron number density is given by the matter density, $n_{e}(x) \simeq \frac{\rho(x)}{2 m_{N}}$. The oscillation probability is given by $P\left(v_{\alpha} \rightarrow v_{\beta}\right)=\left|A_{v_{\alpha} \rightarrow v_{\beta}}\right|^{2}$.

The oscillation probability depends on the density distribution of matter. The energy spectrum of the oscillation probability is distorted by the density profile which is shown in Figure $\mathbb{W}$. However this distortion is so small, and then we need the sufficient statistics for precise reconstruction of the density profile that the neutrino passed through.

In following session, we propose neutrino oscillation tomography with a new neutrino source which enables us to produce a large amount of neutrino pairs. We also propose a reconstruction method to obtain entire distribution by considering the perturbation analytic formula.

\section{Tomography by neutrino pair beam}

We assume the neutrino pair beam as a neutrino source for tomography. This beam is recently proposed in [3], 团]. Pairs of neutrino and anti-neutrino are produced from circulating ions with the quantum coherent mixture state of electron. Characteristics of the beam are as follows: The beam generates all flavor neutrino pairs. It mainly produces electron type neutrino pairs because the cancellation of the coupling in the case of muon- and tau- neutrino pairs [[]]. The beam has a very high intensity and directivity.

Now we estimates how precisely the density profile can be reconstructed. We assume a toy model that satisfies following conditions. First, we consider a symmetric density distribution because the oscillation probability is degenerate under the transformation $\rho(x) \leftrightarrow \rho(L-x)$ in the 2 flavor case. Second we consider the low energy $\bar{v}_{e} \rightarrow \bar{v}_{e}$ oscillation because of a condition 

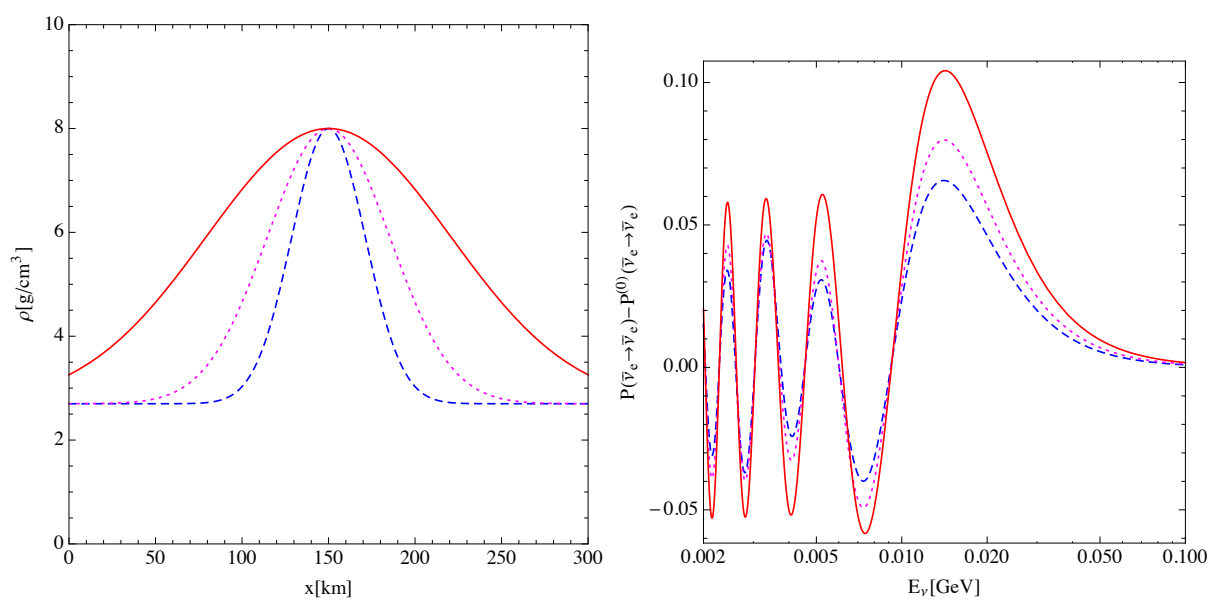

Figure 1: Left panel shows three density profiles. Right panel shows the oscillation probability subtracted by vacuum oscillation in the case of $\Delta m^{2}=7.50 \times 10^{-5} \mathrm{eV}^{2}, \sin ^{2} \theta=0.306$ for the density profiles in left panel.

$\frac{\Delta m^{2}}{2 E_{v}}>\frac{G_{F}}{\sqrt{2} m_{p}} \rho$ which is discussed in [四]. Finally we assume the large detector ${ }^{1}$ with a fiducial volume $V_{d}=10^{5} \mathrm{~m}^{3}$.

We consider a Gaussian-type density distribution with a lump located at the center of the baseline.

$$
\rho(x)=\bar{\rho}+\left(\rho_{l}-\bar{\rho}\right) \exp \left[-\frac{(x-L / 2)^{2}}{D_{l}^{2}}\right],
$$

where $\rho_{l}$ is the density of the lump, $\bar{\rho}$ is a background density, $L$ is a neutrino baseline, $D_{l}$ is the width of the lump. We perform the $\Delta \chi^{2}$ analysis and estimate how precisely $\rho_{l}$ and $D_{l}$ can be measured. In this statistical analysis, we assume $\bar{\rho}=2.7 \mathrm{~g} / \mathrm{cm}^{3}$ which corresponds to the mean value of the Earth's crust. We also assume $L=300 \mathrm{~km}$ and $\Delta \chi^{2}$ is defined by

$$
\Delta \chi^{2}=\sum_{i=1}^{N_{b}} \frac{\left[\left.N\left(E_{i}\right)\right|_{D_{*}, \rho_{*}}-\left.N\left(E_{i}\right)\right|_{D_{l}, \rho_{l}}\right]^{2}}{\sigma^{2}\left(E_{i}\right)}
$$

where $N_{b}=100$ is number of energy bins, $\sigma\left(E_{i}\right)=\sqrt{\left.N\left(E_{i}\right)\right|_{D_{l}, \rho_{l}}} . \rho_{*}$ and $D_{*}$ are arbitrary values of the density and width of the lump, respectively. $N\left(E_{i}\right)$ is an event rate which is given by

$N\left(E_{v}\right) \simeq 4.73 \times 10^{7} \times P\left(\bar{v}_{e} \rightarrow \bar{v}_{e} ; E_{v}\right)\left(\frac{E_{v}}{100 \mathrm{MeV}}\right)^{\frac{5}{2}}\left(\frac{\Delta E_{v}}{1 \mathrm{MeV}}\right)\left(\frac{300 \mathrm{~km}}{L}\right)^{2}\left(\frac{V_{d}}{10^{5} \mathrm{~m}^{3}}\right)\left(\frac{T}{1 \text { year }}\right)$

where we have taken the parameters of the pair beam in [B]. We assume the observation period $T$ is one year. We consider only the statistic error and neglect the systematic errors in this analysis.

The result of this analysis is shown in Figure $\square$. It is seen that the neutrino pair beam can provide a powerful source for the measurement of the density profile. It can probe the lump at the

\footnotetext{
${ }^{1} \mathrm{We}$ assume the inverse beta decay process for neutrino detection. The energy threshold is $1.806 \mathrm{MeV}$ and we took $2 \mathrm{MeV}$ as threshold. We also assume $\Delta E_{V} \sim 1 \mathrm{MeV}$ as energy resolution.
} 


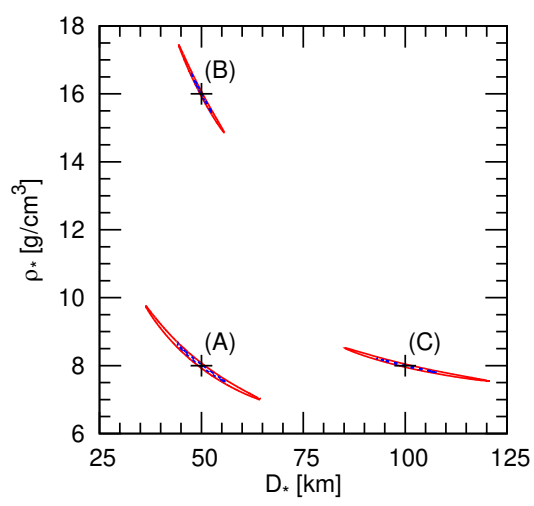

Figure 2: The contour of $\Delta \chi^{2}$ in $\rho_{*}$ and $D_{*}$. Blue dashed line shows $\Delta \chi^{2}=2.3(1 \sigma$ level $)$ and Red line shows $\Delta \chi^{2}=11.83$ (3 $\sigma$ level). The case (A) is $\rho_{l}=8.0 \mathrm{~g} / \mathrm{cm}^{3}, D_{l}=50 \mathrm{~km}$, the case (B) is $\rho_{l}=$ $16.0 \mathrm{~g} / \mathrm{cm}^{3}, D_{l}=50 \mathrm{~km}$ and the case (C) is $\rho_{l}=8.0 \mathrm{~g} / \mathrm{cm}^{3}, D_{l}=100 \mathrm{~km}$.

$1 \sigma$ level as
(A) $D_{*}=50_{-5.9}^{+5.9} \mathrm{~km}$ and $\rho_{*}=8.0_{-0.48}^{+0.62} \mathrm{~g} \mathrm{~cm}^{-3}$,
(B) $D_{*}=50_{-2.4}^{+2.5} \mathrm{~km}$ and $\rho_{*}=16_{-0.53}^{+0.58} \mathrm{~g} \mathrm{~cm}^{-3}$,
(C) $D_{*}=100_{-7.1}^{+8.2} \mathrm{~km}$ and $\rho_{*}=8.0_{-0.21}^{+0.22} \mathrm{~g} \mathrm{~cm}^{-3}$.

Next we propose a method to reconstruct the density profile from the energy spectrum of the neutrino oscillation. Our method is summarized as follow: First, we discretize the neutrino baseline into $N_{L}$ segments and we take $N_{L}=60$. Second, we assign the matter densities for those segment as free parameters $\rho_{j}$ and we assume that the each density is constant within each segment. Third, we also divide the energy range into the $N_{E}$ parts, and define the $\chi^{2}$ function.

$$
\chi^{2}=\sum_{i=1}^{N_{b}} \frac{\left[N^{\mathrm{obs}}\left(E_{i}\right)-N^{\mathrm{th}}\left(E_{i}\right)\right]^{2}}{\sigma^{2}\left(E_{i}\right)}
$$

where $\sigma\left(E_{i}\right)=\sqrt{N^{\mathrm{obs}}\left(E_{i}\right)}$. Finally, we determine density profile by minimizing the $\chi^{2}$ function by comparing the observational data $N^{\mathrm{obs}}\left(E_{i}\right)$ with given original profile $\rho(x)$ and the theoretical prediction $N^{\text {th }}\left(E_{i}\right)$ with unknown parameter $\rho_{j}$. Now we introduce the analytic formula of oscillation probability in $N^{\text {th }}\left(E_{i}\right)$. The analytic formula is derived from the perturbation of the effective potential and we include the correction up to the second order perturbation in this analysis. The detail is found in [四].

The results are shown in Figure 3 and $⿴$. As one can see, the structure of the lump can be reconstructed in the case of method including up to second order perturbation formula. At points near the $x=0$ and $L$, the reconstructed precision is worse than other points. This is because the oscillation probabilities by matter effect at $x=0$ and $L$ become zero. The other details are discussed in [四].

\section{Conclusion}

We investigated the neutrino oscillation tomography. It is found that the neutrino pair beam can produce a large amount of neutrinos. This is an important advantage for the neutrino oscillation 

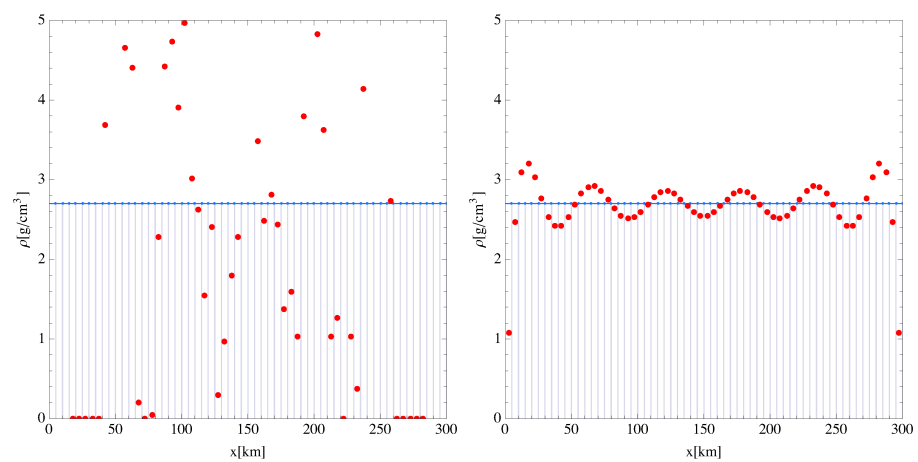

Figure 3: The result of reconstruction : Blue line shows original density profile $\left(\bar{\rho}(x)=2.7 \mathrm{~g} / \mathrm{cm}^{3}\right)$ and red dot shows reconstructed density profile. Left side shows result with up to 1 st order perturbation formula and right side shows results with up to 2 nd order perturbation formula.
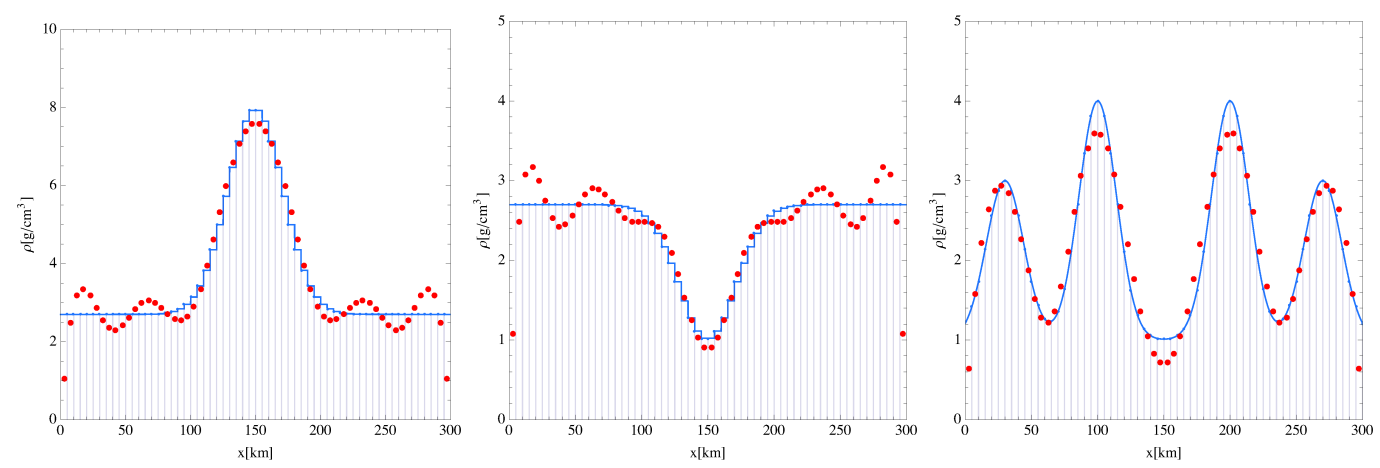

Figure 4: The result of reconstruction with up to the second order perturbation formula : Blue line shows original density profile and red dot shows reconstructed density profile. Left side shows result assuming $\left(\rho_{l}=8.0 \mathrm{~g} / \mathrm{cm}^{3}, D_{l}=30 \mathrm{~km}\right)$, center shows result assuming $\left(\rho_{l}=1.0 \mathrm{~g} / \mathrm{cm}^{3}, D_{l}=30 \mathrm{~km}\right)$ and right side shows complicated density profile.

tomography because the neutrino tomography requires a high neutrino event rate. Thus the neutrino pair beam is powerful tool for the precise probe of the Earth's interior structure. We then propose the reconstruction method for the density distribution based on the analytic formula of oscillation probability. As future tasks, we have to consider more realistic setup. For example, we have to consider the realistic 3 flavor oscillation, the systematic uncertainty of an oscillation experiment, and realistic density distribution of the Earth.

\section{References}

[1] T. Asaka, H. Okui, M. Tanaka and M. Yoshimura, Phys. Lett. B 785 (2018) 536 doi:10.1016/j.physletb.2018.09.004 [arXiv:1805.10793 [hep-ph]].

[2] W. Winter, Earth Moon Planets 99 (2006) 285 doi:10.1007/s11038-006-9101-y [physics/0602049].

[3] M. Yoshimura and N. Sasao, Phys. Rev. D 92 (2015) no.7, 073015 [arXiv:1505.07572 [hep-ph]].

[4] M. Yoshimura and N. Sasao, Phys. Rev. D 93 (2016) no.11, 113018 [arXiv:1512.06959 [hep-ph]].

[5] T. Asaka, M. Tanaka and M. Yoshimura, Phys. Lett. B 760 (2016) 359 [arXiv:1512.08076 [hep-ph]]. 